\title{
Coma morphology and dust emission pattern of comet C/2020 F3 (NEOWISE)
}

\author{
F. Manzini ${ }^{1 \star}$, V. Oldani ${ }^{1}$, P. Ochner ${ }^{2,3}$, E. Barbotin ${ }^{4}$, L. R. Bedin ${ }^{2}$, R. Behrend ${ }^{5}$, \\ and G. Fardelli ${ }^{6}$ \\ ${ }^{1}$ Stazione Astronomica di Sozzago, Cascina Guascona, I-28060 Sozzago (Novara), Italy \\ ${ }^{2}$ INAF-Osservatorio Astronomico di Padova, Vicolo dell'Osservatorio 5, I-35122 Padova, Italy \\ ${ }^{3}$ Department of Physics and Astronomy-University of Padova, Via F. Marzolo 8, I-35131 Padova, Italy \\ ${ }^{4}$ GVO, F-16250 Etriac, France \\ ${ }^{5}$ Geneva Observatory, CH-1290 Sauverny, Switzerland \\ ${ }^{6}$ Via Oberdan 81A, I-64100 Ascoli Piceno, Italy
}

Accepted 2021 June 14. Received 2021 June 8; in original form 2021 February 18.

\begin{abstract}
The recent close approach of comet C/2020 F3 (NEOWISE) allowed us to study the morphology of its inner coma. From the measurement of the dust ejection velocity on spiral structures expanding around the nucleus, we estimated a mean deprojected expansion velocity $V_{\mathrm{d}}=1.11 \pm 0.08 \mathrm{~km} \mathrm{~s}^{-1}$. Assuming that a new shell formed after every rotation of the comet, a rotation period of $7.8 \pm 0.2$ hours was derived. The spin axis orientation was estimated at RA $210^{\circ} \pm 10^{\circ}$, Dec. $+35^{\circ} \pm 10^{\circ}$. The coma morphology appears related to two strong, diametrically opposite emissions located at mid-latitudes on the nucleus. A qualitative modelling of the coma produced consistent results with a wide range of dust sizes $(0.80$ to $800 \mu \mathrm{m})$, with inversely correlated densities $\left(0.003\right.$ to $\left.3.0 \mathrm{~g} \mathrm{~cm}^{-3}\right)$. Images taken with $V j$ and $r$-Sloan filters showed a greater concentration of dust in the first two shells, and an increasing density of radicals emitting in the $B$ and $V$ band-passes from the third shell outwards. Striaelike structures in the tail suggest that dust particles have different sizes.
\end{abstract}

Key words: comets: general - comets: individual (C/2020 F3 [NEOWISE])

\section{INTRODUCTION}

Comet C/2020 F3 was discovered on 2020 March 27, in the context of the NEOWISE program of the NASA's Wide-field Infrared Survey Explorer (WISE) space telescope. At that time the comet was located 2.0 AU from the Sun and 1.7 AU from Earth, appearing as an object of 18th magnitude. C/2020 F3 reached its perihelion on 2020 July 3, at a distance of only 0.29 AU from the Sun. Its closest approach to Earth occurred on 2020 July 23, at a distance of 0.69 AU, when it was at $0.64 \mathrm{AU}$ from the Sun. The comet reached its maximum brightness just after perihelion at magnitude 0.5, becoming the brightest comet visible from the Northern hemisphere after comet Hale-Bopp in 1996 and comet McNaught in 2006.

The extremely favourable conditions of observation and the remarkable activity shown by this comet allowed us to carry out an in-depth study of the phenomena occurring in the inner coma.

\footnotetext{
* E-mail: manzini.ff@aruba.it
}

\section{OBSERVATIONS AND DATA ANALYSIS}

\subsection{Observing instruments}

The CCD images used in this work were collected by our team over forty-two observing sessions from 2020 July 7 to September 3, with telescopes ranging in diameter from 0.3 to $1.82 \mathrm{~m}$. The telescopes used have a spatial resolution between 0.25 and $0.89 \mathrm{arcsec} / \mathrm{pixel}$; the physical resolution in the collected images ranges approximately between 270 and $700 \mathrm{~km} /$ pixel on the sky plane at the distance of the comet.

The full list of images used for this work is provided in Table 1. In the first column the date and mean time (UT) of the observation are shown. In the second column the telescopes used for observations are listed, together with the relevant IAU-MPC (Minor Planet Center www.minorplanetcenter.net/iau/mpc.html) code, which allows to trace the geographic position of the telescopes on the globe. The GVO observatory located in Etriac $\left(45.52626^{\circ}\right.$, $0.02444^{\circ}$, France), is awaiting the IAU-MPC code. The third column shows the sensors used, which equip the following CCD cameras: Sbig-ST8 at site C10, QHY367C at San Gi- 
acomo, Moravian G4-16000 at GVO, Moravian G3-16200 at site A12, Moravian G4-16000 and AFOSC at site 098. The fourth column shows the resolution of each telescope in arcsec/pixel. In the fifth column the filters used during the observations are indicated: with $I, B, V, R$ we mean filters in the Johnson-Cousins bands, with $u, r, i, g, z$ we mean the respective filters in the Sloan bands. Finally, the last column indicates the number of exposures within each observation night and the integration time of each single image.

\subsection{Data reduction}

The data reduction was done using standard IRAF tasks for the images taken at the Asiago (INAF-OAPD) observatory. The data taken at the GVO and IAU-MPC A12 sites were reduced with the Prism and AstroArt 7.0 softwares, respectively (http://www.prism-astro.com and http://www.msb-astroart.com).

Master bias and dark frames were created nightly, then subtracted from the raw images. Depending on the availability of twilight flats, we median combined sky flats frames (at GVO and IAU-MPC A12) or dome flats frames (at IAUMPC 098) to create a single master flat frame for each night. Each single flat frame was reduced with the corresponding master bias and master dark. Finally, each bias- and darksubtracted original image was divided by the master flat frame.

\subsection{Data analysis: coma}

In order to perform a morphological analysis of the comet's inner coma, where it is easier to see structures due to the outflow of gases and dust from active sources on the nucleus, all images were processed with ASTROART 7.0 applying several algorithms: median subtraction, division by $1 / \rho$, radial shift alone with different radii, Larson-Sekanina with angular shift $=20^{\circ}$ and $\mathrm{r}=5$-pixel radial shift (Larson \& Sekanina 1984; Samarasinha, Martin \& Larson 2013), all centred on the optocentre of the comet's nucleus. The comparison between the results of the above methods showed that the highlighted details were always the same and in the same place. The latter process is the one that provided the highest signal, therefore we used it as the basic algorithm for our analyses, always using the other spatial filters as a control.

To fully characterise the inner coma features and to verify their evolution as observed in our images over several observation nights, we then performed a modelling of the inner coma using a proprietary software (FASE 6, by P. Pellissier) specifically designed to reproduce Earth-based observations of the dust coma structures (Manzini et al. 2016). To this purpose, the first element that is needed is the position of the comet with respect to the Sun and the observer. This is easily calculated from the orbital elements of the comet (available in the JPL/HORIZON website http://ssd.jpl.nasa.gov/horizons.cgi). The other parameters needed to run the model are the properties of the dust particles (albedo, size, density, ejection velocity), and those of the nucleus (spin axis orientation, rotation period and position of the active sources). The orientation of the spin axis is the most difficult to obtain, but it is one of the key parameters for the simulation. It was estimated from

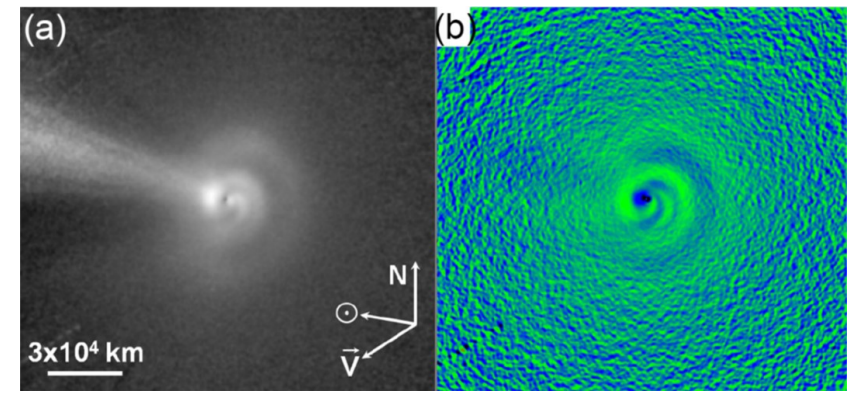

Figure 1. (a) Image of 2020 July 26, processed with a LarsonSekanina filter with a radial shift to enhance the visibility of the morphological structures in the inner coma. Nearly full spirals are visible around the optocentre of comet C/2020 F3, which indicates that the spin axis was directed almost towards the Earth. (b) The spirals, originating from two distinct active sources, are shown more clearly in false colours. North, V and antisolar vectors are indicated. The angle between the observer and the comet orbital plane (Plang) was $-81^{\circ}$.

a first analysis of the coma structures orientations (Vincent, Boehnardt \& Lara 2010), and gradually refined based on the hypothesised position of the active sources on the nucleus and of their associated emissions, as well as on the effect of the insolation on the active sources and of the radiation pressure on the emitted dust. A trial-and-error procedure was applied until a plausible orientation of the axis, compatible with a realistic reproduction of the features visible in the processed images, was achieved.

Finally, to verify the correctness of the estimated position of the spin axis, we made some models of the nucleus with the software STARRY Night PP v. 5.8.4 (Simulation Curriculum Corp., Minnetonka, MN (USA), http://www.simulationcurriculum.com). The model reproduces the geometric conditions of observation of the cometary nucleus from Earth by entering the orbital parameters of the comet and the assumed direction of the spin axis for each of the dates considered.

\subsection{Data analysis: tail}

To highlight the structure of the tail, particularly the presence of striae, synchrones and syndines, we applied a gradient operator to the original wide-field images, which produces an "embossed effect" that increases the visibility of any existing vertical and horizontal brightness gradients. The algorithm is given by the formula:

$$
I^{\prime}(x, y)=I(x, y)-I(x-d x, y-d y)
$$

The resulting image is displayed with a linear transfer function.

We successively compared the findings on the processed images with a two-dimensional numerical model of the general structure of the tail obtained by means of the Finson-Probstein equation (Vincent 2014), using a web-based tool (COMET TOOLBox by J.B. Vincent, http://www. comet-toolbox.com). 
Table 1. Data used in this work.

\begin{tabular}{|c|c|c|c|c|c|}
\hline Date (UT) & Telescope @MPC code & $\mathrm{CCD}$ & arcsec/pixel & Filter & $\mathrm{N} \times$ ExpTime \\
\hline 2020-07-08T02:51* & reflector $0.3 \mathrm{~m} @ \mathrm{C} 10$ & KAF-1603me & 1.63 & $I$ & $10 \times 2 \mathrm{~s}$ \\
\hline 2020-07-10T23:59* & reflector $0.3 \mathrm{~m} @ \mathrm{C} 10$ & KAF-1603me & 1.63 & $I$ & $10 \times 6 \mathrm{~s}$ \\
\hline 2020-07-11T02:04* & refractor 0.1m @S. Giacomo (IT) & IMX 094c & 2.65 & None & $3 \times 60 \mathrm{~s}$ \\
\hline 2020-07-18T16:10 & ob.85mm @Gobi Desert & Canon $\mathrm{ff}$ & 5.8 & None & $40 \times 5 \mathrm{~s}$ \\
\hline 2020-07-21T20:20 ${ }^{\dagger}$ & Copernico 1.82m @098 & E2V CCD42-40 DD & 0.25 & BVrigz & $18 \times 10 \mathrm{~s}$ \\
\hline 2020-07-22T21:20* & reflector $0.5 \mathrm{~m} @ \mathrm{GVO}(\mathrm{FR})$ & KAF-16803 & 0.54 & None & $100 \times 20 \mathrm{~s}$ \\
\hline 2020-07-24T20:56* & reflector 0.5m @GVO (FR) & KAF-16803 & 0.54 & None & $45 \times 30 \mathrm{~s}$ \\
\hline 2020-07-25T20:05 ${ }^{\dagger}$ & Schmidt 67/92@098 & KAF-16803 & 0.87 & $u B V r i$ & $34 \times 50 \mathrm{~s}$ \\
\hline 2020-07-26T21:10* & reflector 0.5m@GVO (FR) & KAF-16803 & 0.54 & None & $100 \times 30 \mathrm{~s}$ \\
\hline 2020-07-28T21:00* & reflector $0.5 \mathrm{~m} @ \mathrm{GVO}(\mathrm{FR})$ & KAF-16803 & 0.54 & None & $75 \times 30 \mathrm{~s}$ \\
\hline 2020-07-31T20:51* & reflector $0.5 \mathrm{~m} @ \mathrm{GVO}(\mathrm{FR})$ & KAF-16803 & 0.54 & None & $30 \times 80 \mathrm{~s}$ \\
\hline 2020-08-03T21:00* & reflector $0.5 \mathrm{~m} @ \mathrm{GVO}(\mathrm{FR})$ & KAF-16803 & 0.54 & None & $75 \times 30 \mathrm{~s}$ \\
\hline 2020-08-05T21:00* & reflector 0.5m @GVO (FR) & KAF-16803 & 0.54 & None & $75 \times 30 \mathrm{~s}$ \\
\hline 2020-08-09T19:53 ${ }^{\dagger}$ & Schmidt 67/92@098 & KAF-16803 & 0.87 & $B r$ & $10 \times 30 \mathrm{~s}$ \\
\hline 2020-08-09T20:07* & reflector $0.4 \mathrm{~m} @ \mathrm{~A} 12$ & KAF-16200 & 0.86 & $B V R$, None & $80 \times 30 s$ \\
\hline 2020-08-18T20:15* & reflector $0.4 \mathrm{~m} @ \mathrm{~A} 12$ & KAF-16200 & 0.86 & $B V R$, None & $10 \times 180 \mathrm{~s}$ \\
\hline
\end{tabular}

* this material is available at our repository:

https://web.oapd.inaf.it/bedin/files/PAPERs_eMATERIALs/NEOWISE_C2020F3_MNRAS/

$\dagger$ this material is available at: http://archives.ia2.inaf.it/aao/
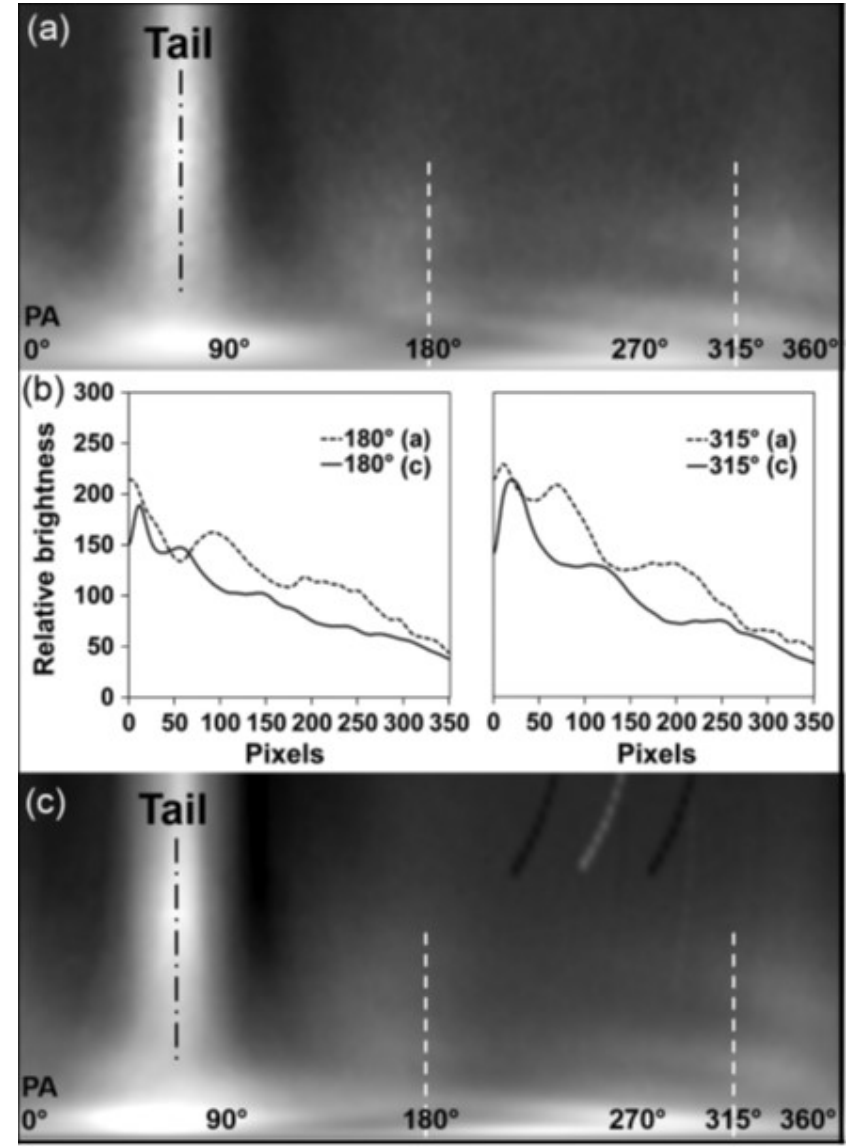

Figure 2. (a and c:) Polar projections, centred on the optocentre of the false cometary nucleus, of two images of 2020 July 26 , processed as in Fig. 1, taken with 1-hour time interval. The projections show the development and expansion of the spirals. (b) The vertical photometric profiles at $\mathrm{PA} 180^{\circ}$ and $315^{\circ}$ provide the precise distance of each shell from the nucleus (in pixels).

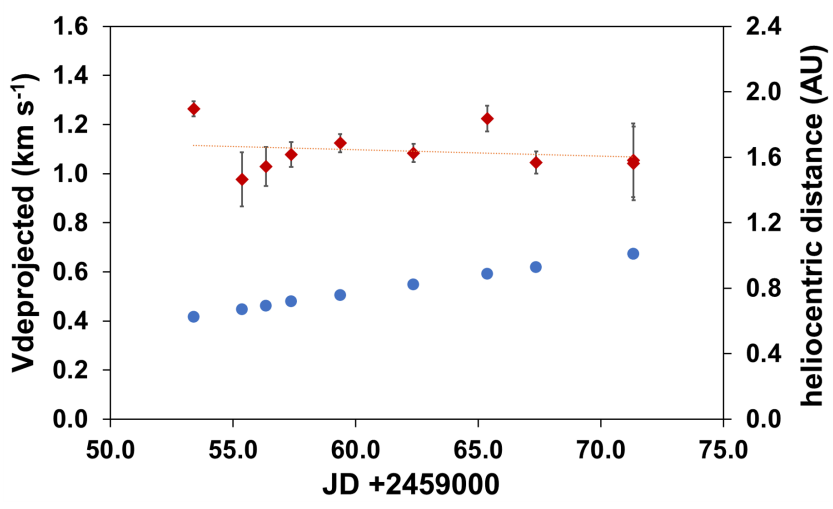

Figure 3. The mean ( \pm standard deviation) deprojected expansion velocity (diamonds) of the spiral-shaped structures measured on ten observing sessions is shown in relation to the heliocentric distance (dots). The dotted line shows the linear trend of the expansion velocity.

\section{RESULTS}

\subsection{Coma dust structures}

Bow-shaped structures and haloes, which developed between PA $180^{\circ}$ and PA $290^{\circ}$, subjected to strong radiation pressure, were visible on the first series of images obtained on 2020 July 10 (comet at $\mathrm{r}=0.35$ AU). Subsequently, these structures turned into spiral segments with origin on the cometary nucleus (Manzini et al. 2020), and became almost full spirals around July 26 (Fig. 1). By August 15 they had almost vanished and in the first days of September there was no longer trace of them.

We estimated the dust ejection velocity on two CCD images taken at least 1 hour apart on ten observing sessions from 2020 July 22 to August 9 (Table 2). All images were first transformed into polar maps centred on the optocentre of the nucleus in order to determine the position of the dust waves with the highest possible precision. We measured 
Table 2. Date, JD, distance from the Sun (r), distance from the Earth $(\Delta)$, and measured mean deprojected expansion velocity $\left(V_{\mathrm{d}} \pm\right.$ standard deviation) in the observing sessions.

\begin{tabular}{lcccr}
\hline \hline Date (UT) & JD & $\mathrm{r}(\mathrm{AU})$ & $\Delta(\mathrm{AU})$ & $V_{d}\left(\mathrm{~km} \mathrm{~s}^{-1}\right)$ \\
\hline $2020-07-08.10$ & 2459038.60 & 0.3256 & 0.9961 & - \\
$2020-07-11.00$ & 2459041.50 & 0.3718 & 0.8980 & - \\
$2020-07-11.08$ & 2459041.58 & 0.3736 & 0.8948 & - \\
$2020-07-18.67$ & 2459049.17 & 0.5322 & 0.7203 & - \\
$2020-07-21.85$ & 2459052.35 & 0.6033 & 0.6939 & - \\
$2020-07-22.89$ & 2459053.39 & 0.6257 & 0.6919 & $1.264 \pm 0.030$ \\
$2020-07-24.86$ & 2459055.36 & 0.6703 & 0.6965 & $0.977 \pm 0.110$ \\
$2020-07-25.84$ & 2459056.34 & 0.6925 & 0.7029 & $1.029 \pm 0.079$ \\
$2020-07-26.87$ & 2459057.37 & 0.7195 & 0.7119 & $1.078 \pm 0.051$ \\
$2020-07-28.87$ & 2459059.37 & 0.7585 & 0.7371 & $1.124 \pm 0.037$ \\
$2020-07-31.86$ & 2459062.36 & 0.8234 & 0.7899 & $1.084 \pm 0.037$ \\
$2020-08-03.86$ & 2459065.36 & 0.8872 & 0.8568 & $1.224 \pm 0.053$ \\
$2020-08-05.86$ & 2459067.36 & 0.9292 & 0.9073 & $1.046 \pm 0.045$ \\
$2020-08-09.82$ & 2459071.32 & 1.0105 & 1.0167 & $1.054 \pm 0.150$ \\
$2020-08-09.83$ & 2459071.33 & 1.0105 & 1.0167 & $1.041 \pm 0.150$ \\
$2020-08-18.80$ & 2459080.30 & 1.1889 & 1.2922 & \\
\hline
\end{tabular}

the shell-to-shell distance in pixels in four different points at $45^{\circ}$ intervals from $\mathrm{PA} 180^{\circ}$ to $315^{\circ}$ around the nucleus, averaged the four measures into a single daily value and then converted this distance $D_{\text {projected }}$, projected on the plane of the sky, into kilometres (Fig. 2).

The expansion velocity of these structures was calculated by the simple relation

$$
V_{\text {projected }}=D_{\text {projected }} / \Delta t
$$

where $\Delta t$ is the time difference in seconds between the two images on each day. However, since during the considered time-span the phase angle $\phi$ of the nucleus showed some variation, we also calculated the deprojected distance for each date to correct the corresponding deprojected expansion velocities, using:

$$
D_{\text {deprojected }}=D_{\text {projected }} / \sin \phi
$$

Finally, all the resulting daily values were averaged to obtain a single value for the deprojected expansion velocity $V_{\text {deprojected }}=1.11 \pm 0.08 \mathrm{~km} \mathrm{~s}^{-1}$.

The expansion velocities were essentially steady (or only slightly decreasing) throughout the analysed period (Fig. 3). Moreover, the presence of spiral-shaped structures around the nucleus indicates that during this time-span the spin axis of the comet was directed almost towards the Earth (Schleicher \& Farnham 2004). Therefore, the average of all

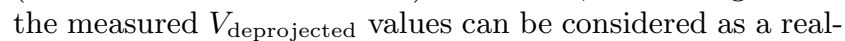
istic estimate of the ejection velocity of dust from the active areas on the cometary nucleus.

\subsection{Rotation period}

We made an estimation of the comet's rotation period on the images taken on 2020 July 26, which showed shells originating from the same active source with the best resolution (Fig. 1). Assuming that a new shell formed after every rotation of the comet, the rotation period of the nucleus $\mathrm{p}$ is simply related to the expansion velocity $\mathrm{V}$ of the shells by the relationship:

$$
p=D_{\text {deprojected }} \cdot V_{\text {deprojected }}^{-1} \text { (Braunstein et al. 1997) }
$$

Being the average shell-to-shell relative distance $\sim 30500 \mathrm{~km}$ and the deprojected expansion velocity $V_{\text {deprojected }}=1.11$ $\mathrm{kms}^{-1}$, a rotation period of $7.8 \pm 0.2$ hours could be derived, consistent with the 7.6 hours reported by Drahus et al. (2020).

\subsection{Modelling of the inner coma}

In order to better characterise the inner coma structures observed on the images and to determine the main properties of the nucleus, we ran a computer model of the inner coma with our proprietary software FASE 6 . To this purpose, a number of parameters are needed (as described in Section $2.3)$.

The previously established parameters, deduced from our measurements were:

- for the rotation period, a value of 7.8 hours;

- for the dust ejection velocity, the value of $1.11 \mathrm{~km} \mathrm{~s}^{-1}$, estimated as described above, was initially entered, although slightly lower values were also tested, in line with the observations (Fig. 3).

Other unknown parameters were determined as follows:

- for the physical parameters of the dust particles, diametres between 0.80 and $800 \mu \mathrm{m}$, with albedo set at 0.03 , were tested in the model. All these particle sizes produced consistent results in the model for inversely correlated values of density between 0.003 and $3.0 \mathrm{~g} \mathrm{~cm}^{-3}$, the smallest particles having higher density and the largest particles having lower density. However, the model does not allow to perform a quantitative analysis and to determine the exact dust size distribution.

- For the latitude and longitude positions of the active source(s) on the nucleus (assumed to be spherical) a trialand-error procedure was followed, moving each potentially identified source by steps of five degrees in both coordinates, and running the model applying a variable number of rotations of the nucleus, until it returned the best approximation of the appearance of the features on the Earth-based processed CCD images. This approach enabled us to relate the complex phenomena observed on the images to the presence of at least two active sources located at approximately $\left( \pm 5^{\circ}\right)$ the following positions on the cometary nucleus:

(i) latitude: $40^{\circ} \mathrm{N}$; longitude: $0^{\circ}$ (arbitrary, taken as reference for the other sources)

(ii) latitude: $65^{\circ} \mathrm{N}$; at $225^{\circ}$ longitude from source (i).

Considering the geometric conditions of the observation and the parameters indicated, these active sources have been identified as those responsible for the formation of the spirals expanding from the nucleus.

A possible third, additional source was also hypothesised, located in this position:

(iii) latitude: $80^{\circ} \mathrm{N}$; at $45^{\circ}$ longitude from source (i);

because of its position in latitude on the cometary nucleus, this third source produced no spirals or shells, but only an almost continuous outflow straight in the direction of the tail at a short distance from the nuclear regions. 

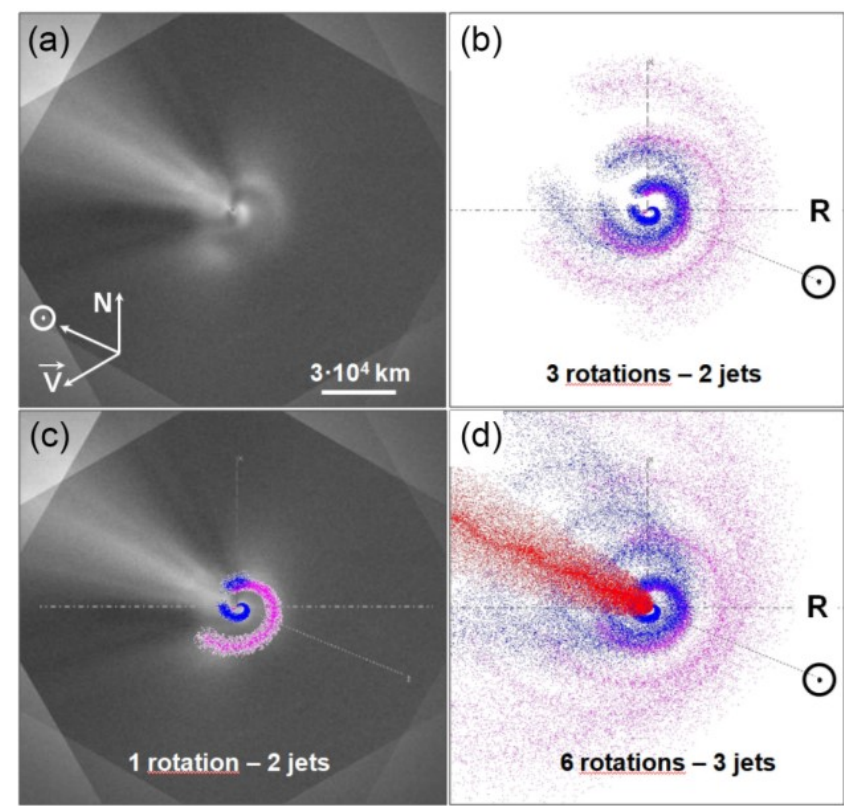

Figure 4. Modelling of the inner coma structures compared with CCD images of 2020 July 24, processed with a Larson-Sekanina filter with a radial shift(a). The model shows the best agreement with the observed morphology when three active sources are considered on the rotating nucleus. In panel (b) two sources and three rotations are considered to better show the inner coma. (c) The result of a single rotation with two sources is superimposed to the CCD image. (d) A third, near-polar jet has been added to the model and six rotations of the nucleus have been applied. North, V and solar or antisolar vectors are indicated. The comet's spin axis is indicated with $\mathrm{R}$. The orientation of the spin axis on this date was estimated at $\mathrm{PA} \sim 270^{\circ}$ with an inclination of $\sim 75^{\circ}$ from the sky plane.

- The direction of the spin axis of the nucleus of comet C/2020 F3 during the observation period was determined at approximately $\mathrm{RA} 210^{\circ} \pm 10^{\circ}$, Dec. $+35^{\circ} \pm 10^{\circ}$, with a prograde rotation of the nucleus.

To best represent the coma structures, the software was set to plot 150 dots at 10 -minute intervals over one to six rotations, such as to reproduce a virtually continuous outflow, and assigning a different colour to each identified source, in order to distinguish the relevant dust emissions between each other.

An example of the resulting model, in comparison with the respective CCD image, is shown in Fig. 4, where the jets (i) and (ii) are shown in violet and blue, respectively.

\subsection{Differences between images in $\mathrm{Vj}$ and $\mathrm{r}$-Sloan filters}

On some of the observation dates, the presence of two or more shells made it possible, by comparing immediately consecutive images in the $\mathrm{Vj}$ and r-Sloan band, to estimate the distance at which the ratio between gas and the conveyed dust becomes predominant in favour of the former or the latter.

Images taken consecutively on 2020 July 21 with the 1.82m Copernico telescope (INAF-Asiago Astrophysical Ob-

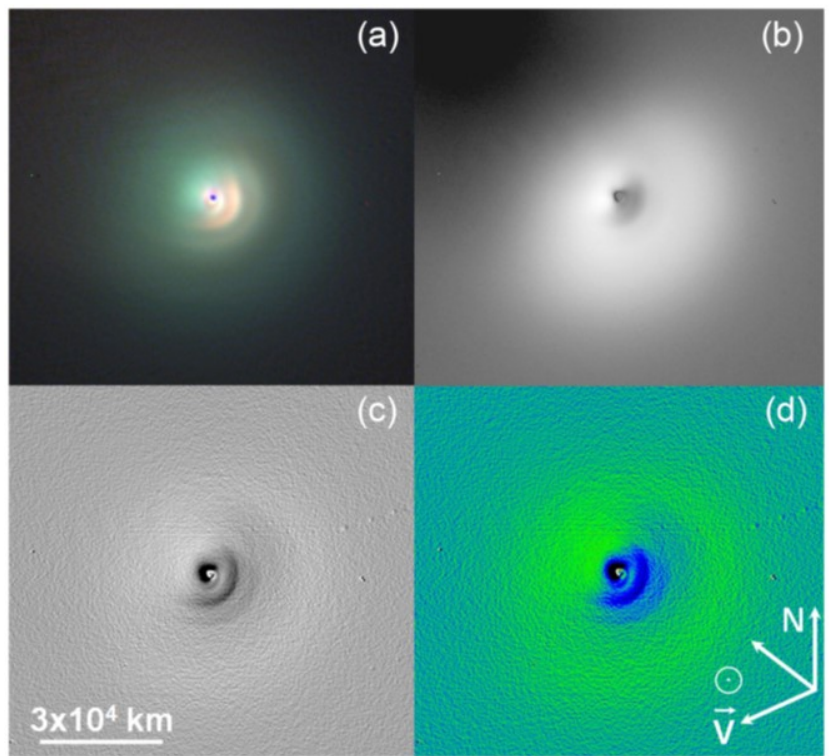

Figure 5. Images of 2020 July 21, taken with the 1.82-m Copernico telescope at the Asiago Astrophysical Observatory. (a) Composite $(\mathrm{Bj}, \mathrm{Vj}, \mathrm{r}-\mathrm{Sloan})$ image calibrated in each colour channel. The dust component, best shown by the r-Sloan filter, is evident in the subsolar sector of the first two shells. The coma appears dominated by the $\mathrm{V}$ emissions. The black dot at centre shows the position of the optocentre of the nucleus. (b-d) Result of dividing the $\mathrm{Vj}$ image by the r-Sloan image: the dust component (darker) is spread relatively close to the nucleus, before being affected by the radiation pressure; the dust tail appears in PA $45^{\circ}$. (c and d) Same image as in panel (b), after applying a radial shift of 3 pixels to enhance the differences in brightness around the nucleus, shown in grey shades and false colours.

servatory) were processed to assess the difference in the intensity level of the shells between the data in $\mathrm{Vj}$ and in r-Sloan (Fig. 5).

The same original $\mathrm{Vj}$ and $\mathrm{r}$-Sloan images were also processed by means of a radial shift of 10 pixels to increase the contrast of all those details showing a gradient of brightness with respect to the optocentre, followed by a $1 / \rho$ filter to compensate for the radial fall-off of brightness (Samarasinha, Martin \& Larson 2013). This approach determined a loss of the photometric data, however the images in the two filters have been equally processed and the information regarding the intensity ratio between the gradients of the shell structures was fully preserved. Successively, we traced on both images the brightness profiles for 400 pixels in the sunward direction starting from the optocentre, plotted both curves using an arbitrary scale for the brightness levels and then compared the peak values corresponding to the three shells for each curve (Fig. 6).

The analysis showed that the peak intensity ratio between the first and second shell was:

- $\mathrm{Vj}=1.20$;

- r-Sloan = 1.10;

and the peak intensity ratio between the first and third shell was:

- $\mathrm{Vj}=1.85$;

- $\mathrm{r}-\mathrm{Sloan}=2.19$; 


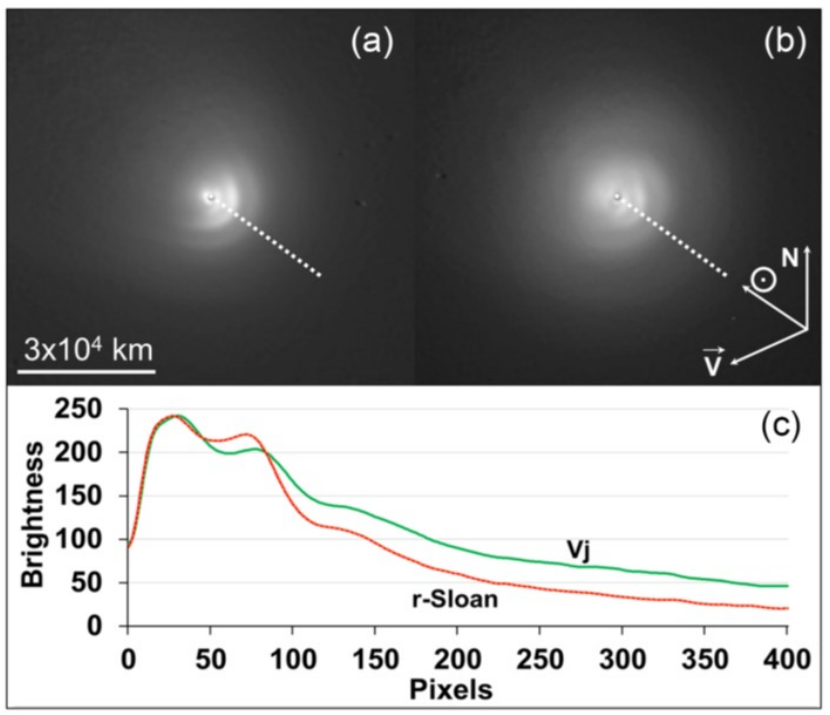

Figure 6. (a and b) Images of 2020 July 21 taken with the 1.82$\mathrm{m}$ Copernico telescope at the Asiago Astrophysical Observatory in broadband r-Sloan and Vj filters. Resolution: $63 \mathrm{~km} /$ pixel. (c) Brightness profile of the $\mathrm{Vj}$ and r-Sloan images traced for 400 pixels $(\sim 27,000 \mathrm{~km}$; dotted line in panels (a) and (b)) from the optocentre (black dot) in the sunward direction. The colour of each line represents the corresponding filter. In order to make a direct comparison of the respective slopes, an appropriate vertical shift was applied to the curves until the peak values corresponding to the first shell were equalized.

The curve related to the brightness profile of the $\mathrm{Vj}$ image showed also that the peak values corresponding to the shells were slightly shifted towards the direction of the sun with respect to the r-Sloan curve. These data indicate that the contribution of the $\mathrm{Vj}$ image was predominant from the third shell outward, while the contribution of the r-Sloan image was predominant in the subsolar sector in the first two shells, which suggests that the dust was more concentrated in that area, before being affected by the radiation pressure.

The shell expansion over 1-hour time span is showed in a tri-colour animation (C2020F3_20200724.mp4, available on-line).

\subsection{Tail structures}

The orientation of the tail appeared to be clearly connected to the anti-solar vector over the entire observation period.

When the comet was between 0.35 AU and 0.55 AU from the Sun (2020 July 10 to July 20), the Earth was far below the comet's orbital plane (between $-40^{\circ}$ and $-70^{\circ}$ ) and the nucleus showed a phase angle of approximately $100^{\circ}$. Therefore, the tail was geometrically projected almost on the plane of the sky, and its appearance was scarcely affected by the position of the comet in relation to the Earth. In this period, striae-like structures, as described by Sekanina \& Farrell (1980) and by Fulle (2004), were observed in the tail, probably resulting from dust fragmentation (Price et al. 2019) or interaction of charged dust with the solar wind (Fulle et al. 2015). The short-term data we have do not provide clear evidence of repetitive emissions of material from different discrete sources on the nucleus, active during the cometary day and quiescent during the night, so alternately exposed to solar radiation as the nucleus rotated (Kharchuk \& Korsun 2010).

A deep enhancement processing of the comet's tail in a wide-field image of comet C/2020 F3 taken on 2020 July 11 highlighted the presence of several striae or streamers and two large bands in the striae probably resulting from the diffusion of dust particles of different sizes over time (Fig. 7).

An animation of the motion of the structures and filaments in the tail is shown in the supplementary material (C2020F3_20200722.mp4, available on-line).

In the tail the only significant forces affecting the dusts grain trajectories are the solar gravity and radiation pressure. These forces work in opposite directions and depend on the square of the heliocentric distance. The motion of dust follows the Finson-Probstein equation

$$
m_{\mathrm{d}} \cdot a_{\mathrm{d}}=F_{\text {grav }}(1-\beta)
$$

where $m_{\mathrm{d}}$ and $a_{\mathrm{d}}$ are the mass and acceleration of the dust particles, $F_{\text {grav }}$ is the solar gravity force, and $\beta$ is the ratio (radiation pressure)/(solar gravity), and is inversely proportional to the size of the grains for particles larger than $1 \mu \mathrm{m}$ (Bohren \& Huffman 1983).

A bi-dimensional numerical model drawn according to the Finson-Probstein equation for the date of 2020 July 11 fits well with the direction and length of the tail observed on CCD images taken on the same date for values of $\beta$ between 0.1 and 0.7. Assuming a single value of the dust bulk density of $1.0 \mathrm{~g} \mathrm{~cm}^{-3}$ and a dust scattering efficiency of 1.0 , according to the Finson-Probstein equation (3) (in: Finson \& Probstein 1968), it follows that most of the dust particles had diameters approximately between 1.7 and $12 \mu \mathrm{m}$. However, it must be considered that this synchrone-and-syndine model only provides approximate estimates, since it does not take into account the fact that the dust leaves the coma with a non-zero ejection speed.

\section{DISCUSSION}

The applied image processing algorithms are aimed at highlighting local gradients brightness in the coma, at a distance of a few thousands kilometres from the surface of the comet's nucleus, by reducing the brightness due to the isotropic component of emissions. This methodology allows to analyse in detail the collimated structures in the inner coma, however it does not allow to establish their contribution to the total dust emissions.

The complex morphology that we observed in the inner coma of comet C/2020 F3 seems to be related to the presence of at least two active sources located at different latitudes and longitudes on the rotating nucleus (assumed spherical) and to the position assumed by the spin axis, with its North pole constantly exposed to sunlight. Both the geometric conditions of observation from the Earth and the conditions of insolation of the comet's nucleus due to its orbital motion have changed during the observation period. As a result, the orientation of the spin axis showed a slow counter-clockwise rotation, and the appearance of the morphological features of the inner coma changed slightly over time (Fig. 8). 


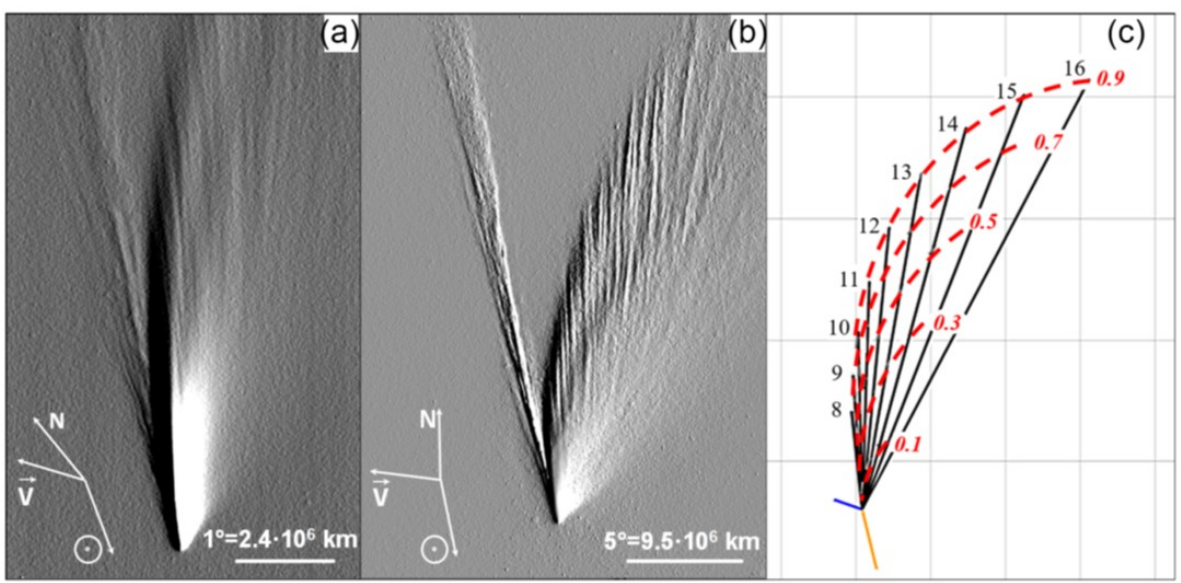

Figure 7. A simple gradient operator was applied to original wide-field images to increase the visibility of any existing vertical and horizontal brightness gradients, such as striae, synchrones and syndines. (a) Image of 2020 July 11.1 UT; UV/IR cut filter. The angle between the Earth and the comet orbital plane (Plang) was $-41^{\circ}$. (b) Image of 2020 July 18.7 by L. Zixuan. Plang: $-62.5^{\circ}$. Distances are in $\mathrm{km}$ on the sky plane. (c) Finson-Probstein diagram plotted on the same date and with approximately the same scale as the image in Panel (b) (intervals between vertical gridlines are of $5^{\circ}$ ). The diagram shows the tail geometry with the development of synchrones (solid lines) over the previous 8 to 16 days and of syndines (red dashed lines) for $\beta$ values of 0.1 to 0.9 . Modified from: Comet Toolbox by J.B. Vincent, http://www.comet-toolbox.com.

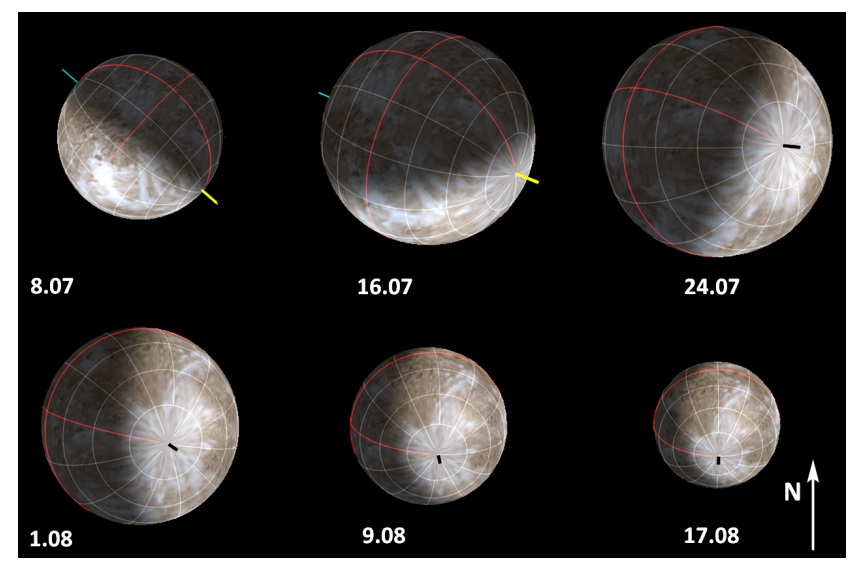

Figure 8. The direction of the spin axis of comet C/2020 F3 as seen from Earth between 2020 July 8 and August 17 is shown in yellow or black on a supposedly spherical nucleus, based on its estimated pole position at RA $210^{\circ}$ and Dec. $+35^{\circ}$. The phase effect is also shown. The minimum inclination of the spin axis with respect to the line of sight from the Earth was reached between late July and mid-August, favouring the observation of almost complete spirals from emissions deriving from the active areas on the nucleus.

The computer model that we have reconstructed shows the best agreement with the features observed on the CCD images, in particular the shell formation, when two almost diametrically opposite sources, characterized by high-speed emission of dust conveyed by gas, are placed at mid-latitudes on the cometary nucleus. Furthermore, the model hypothesised the possible presence of a third active source located in a near-polar position, which was not contributing to any of the features of the inner coma, probably due to a lower emission speed, whereby the dust was more affected by the solar radiation pressure showing only an almost continuous outflow straight in the direction of the tail.

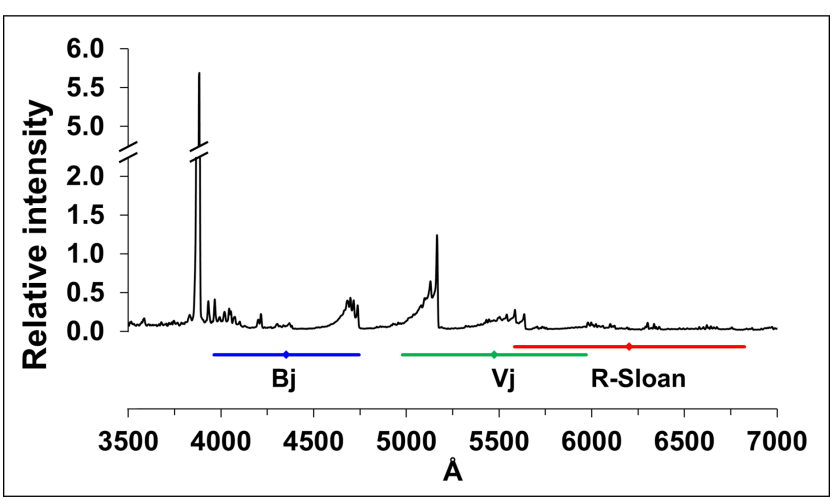

Figure 9. Two spectra were obtained on 2020 August 08 with the 1.22-m Galileo telescope at the Asiago Astrophysical Observatory with an exposure of $600 \mathrm{~s}$ and a resolution of $2.25 \AA$ pixel $^{-1}$. The two calibrated spectra were divided by a solar analogue to offset the contribution of the Sun, then co-added. The emissions of CN at $3880 \AA$ and the $\mathrm{C} 3$ and $\mathrm{C} 2$ Swan-bands are very obvious. Below the spectrum, the respective central wavelength and FWHM of the $\mathrm{Bj}, \mathrm{Vj}$ and $\mathrm{r}-\mathrm{S}$ Sloan filters are shown.

The model revealed that the dust outflow from the identified sources is probably composed of particles in a wide size range, between 0.8 and $800 \mu \mathrm{m}$, compatible with the observations of Rosetta on comet $67 \mathrm{P}$ (Fulle et al. 2015; Rotundi et al. 2015; Della Corte et al. 2015) and with the finding of a significant presence of particles of large size (in the submillimetre range) in the coma by the Giant Metrewave Radio Telescope (Pal, Manna \& Kale 2021). The model showed an inverse correlation between the size of the dust particles and their density. The full dust size distribution is however difficult to determine since the modelling is purely qualitative. In fact, modelling the properties of the dust particles is extremely difficult, as in general only little information about 
the characteristics of the dust grains is available and only one dust size at a time can be entered to run our model.

It should be said that the model only reproduces the spatial disposition of the dust emitted by discrete sources on the nucleus, which may only be partially responsible for the formation of the coma compared to the isotropic release of dust from the sunlit nuclear surface. In addition, the model assumes a spherical nucleus, whereas a complex three-dimensional topography of the nucleus may often be responsible for the development of peculiar coma morphologies (Kramer \& Noack 2015).

The images of the observation session of 2020 July 26 showed the presence of very obvious spiral shells that were most likely arising from the same source, but also of other shells that seemed to originate from a different active area on the cometary nucleus (Fig. 1; see also Fig. S10 and C2020F3_20200726.mp4, available on-line). Indeed, in most of the images taken between mid-July and early August it appears that the some of the observed shells were due to the overlap of the emissions from two different sources, as also confirmed by our modelling (Fig. 4). This feature supports the presence of two strongly emitting sources, almost symmetrical to each other, and it might also be due to a possible slightly different emission speed, and/or of a variability of the emission speed over time, of the two sources.

The value of $1.11 \pm 0.08 \mathrm{~km} \mathrm{~s}^{-1}$ that we found for the expansion speed of the observed structures is higher than those reported for other comets, although it is not unusual. For example, the expansion speed in comet Hale-Bopp was measured to range over time from 0.40 to $1.41 \mathrm{~km} \mathrm{~s}^{-1}$ (Manzini et al. 2001), and $0.67 \pm 0.07 \mathrm{~km} \mathrm{~s}^{-1}$ (Braunstein et al. 1997). Dust speed was calculated to range from 0.20 to $0.45 \mathrm{~km} \mathrm{~s}^{-1}$ in comet 21P/Swift-Tuttle (Jorda, Colas \& Lecacheux 1994; Schulz et al. 1994), comet Levy 1990c (Fulle, Pasian \& Benvenuti 1992), comet $\mathrm{P} / 2004$ A1 (Mazzotta Epifani et al. 2006) and comet Halley's 1910 passage (Larson \& Sekanina 1984). The Giotto probe measured in situ a neutral gas expansion velocity of $0.9 \mathrm{~km} \mathrm{~s}^{-1}$ (Krankowsky et al. 1986). Comet $8 \mathrm{P} /$ Tuttle showed a sunward emission speed of $0.96 \pm 0.03 \mathrm{~km} \mathrm{~s}^{-1}$ (Waniak et al. 2009).

The comet's rotation period was estimated at $\sim 7.8$ hours; this value was derived from the measurement of the shell expansion velocities, which despite was done on images taken in different dates from those used by Drahus et al. (2020), led to comparable results. The measured expansion speeds were essentially stable during the observation period from 2020 July 22 to August 9, with the comet being at a distance of $0.63<\mathrm{r}<1.01 \mathrm{AU}$ from the Sun. This is consistent with a steady rotation period of the comet during this time. Furthermore, the value of the rotation speed that we found was also confirmed by the modelling; in fact, by applying this period, the model made a reproduction of the development of the features of the inner coma very close to those observed on the CCD images according to the number of rotations applied (Fig. 4).

The analysis of the images taken with Johnson-Cousins and Sloan filters made it possible to make a qualitative analysis of the different contribution of dust and gases to the brightness of the coma and of the observed structures. Although the used broadband filters include both gas and dust emissions, in some cases their use can provide some hints on the dust-to-gas ratio. Since the r-Sloan band-pass is centred at $\lambda=6204 \AA$, with an equivalent width of $\sim 1240 \AA$, it should be least affected by possibly present gaseous emissions, as the next strongest bands (i.e., those of C3, C2 and $\mathrm{CN}$ ) are located in the blue to green spectral range (Schulz et al. 2003). Therefore, the comparison of the R-band images with the $\mathrm{V}$ - and B-band images can allow to make a rough qualitative assessment of the dust-to-gas ratio. In the case of comet NEOWISE F3, this hypothesis is confirmed by observations: Schleicher, Knight \& Skiff (2020) reported a lower dust-to-gas ratio than the standard for a comet, and spectroscopic observations (Krishnakumar et al. 2020; Mugrauer \& Bischoff 2020) reported strong emissions of CN and C2, confirmed by a spectrographic analysis performed with the 1.22-m Galileo telescope (INAF-Asiago Astrophysical Observatory - Padua University) on 2020 August 4 (Fig. 9). From these observations we concluded that the dust was more concentrated in the subsolar sector in the first two shells, while the gas component was predominant from the third shell until the edge of the coma (Fig. 6).

\section{CONCLUSIONS}

From our analyses of the CCD images of comet C/2020 F3, and the modelling of its inner coma features, we found the following:

(i) the comet is strongly emitting gas and dust, at high ejection speed, most likely from two different active sources located in almost symmetrical positions at mid-latitudes on the nucleus. A possible third source, located in near-polar position was also hypothesised.

(ii) The comet spin axis is directed at RA $210^{\circ}$, Dec. $+35^{\circ}$, with a prograde rotation. The spin axis was directed towards the Earth during the whole observation period, and spirals and shell-shaped structures, deriving from the active sources, become visible in the inner coma.

(iii) The rotation period is confirmed at $\sim 7.8$ hours. The rotation is stable, occurs on a single axis and is not chaotic.

(iv) The coma is probably composed of a wide range of dust sizes (between 0.80 and $800 \mu \mathrm{m}$ ), inversely correlated with their density values $\left(0.003\right.$ to $\left.3.0 \mathrm{~g} \mathrm{~cm}^{-3}\right)$. The exact dust size distribution is however difficult to determine.

(v) The dust was more concentrated in the subsolar sector in the first two shells, while the gas component was predominant from the third shell to the edge of the coma.

(vi) The $\beta$ values of the dust particles in the tail have been estimated approximately between 0.1 and 0.7 by means of a bi-dimensional Finson-Probstein model.

\section{ACKNOWLEDGMENTS}

This research is based on observations collected at the Copernico and Schmidt telescopes (Asiago, Italy) of INAF Osservatorio Astronomico di Padova. We would like to thank Lina Tomasella, Jean François Soulier and Lin Zixuan for sharing important images and data for the realization of this article, and Gabriele Cremonese for the valuable advice.

We would like to acknowledge the anonymous reviewer whose helpful comments significantly improved the quality of this manuscript. 


\section{DATA AVAILABILITY}

The images collected with the Copernico and Schmidt telescopes at the Asiago Observatory are directly downloadable from the INAF institutional archive interface. The wide-field image of 2020 July 18 was provided by Lin Zixuan by permission. It will be shared on request to the corresponding author with permission of Lin Zixuan. All the remaining data underlying this article are available at our group repository at INAF. The full list of images and the URL of the corresponding repository are shown in Table 1.

\section{REFERENCES}

Bohren C.F., Huffman D.R., 1983, Absorption and Scattering of Light by Small Particles. Wiley, New York

Braunstein M. et al., 1999, Earth Moon Planets, 78, 219

Della Corte V. et al., 2015, A\&A, 583, A13

Drahus M., Guzik P. Stephens A. Howell S. B. Zola S. Sabat M. Reichart D. E. 2020, Astron. Telegram, 13945, 1

Finson M.J., Probstein R.F., 1968, ApJ 154, 327

Fulle M., 2004, in Festou M. C., Keller U., Weaver H. A., eds, Comets II. Univ. Arizona Press, Tucson, Arizona, USA, p. 565.

Fulle M., Pasian F., Benvenuti P., 1992, Ann. Geophys., 10, 145

Fulle M., et al., 2015, ApJ 802, L12

Jorda L., Colas F., Lecacheux, J., 1994, Planet. Space Sci., 42, 699

Kharchuk S.V., Korsun P.P., 2010, Kinematics Phys. Celest. Bodies, 266, 322

Kramer T., Noack M., 2015, ApJ 813, L33

Krankowsky, D.L.P. et al., 1986, Nature 321, 326

Krishnakumar A., Angchuk D., Venkataramani K., Ganesh S., Sahu D.K., Sivarani T., Unni A., 2020, Astron. Telegram, 13897, 1

Larson S.M., Sekanina Z., 1984, AJ 89, 571

Manzini F., Crippa R., Oldani V., Guaita C., 2001, Earth Moon Planets 87,73

Manzini F., Oldani V., Behrend R., Ochner P., Baransky A., Starkey D., 2016, Planet. Space Sci., 129, 108

Manzini F., Ochner P., Oldani V., Bedin L.R., 2020, Telegram, 13884,1

Mazzotta Epifani E., Palumbo P., Capria M.T., Cremonese G., Fulle M., Colangeli L., 2006, A\&A 460, 935

Mugrauer M., Bischoff R., 2020, Astron. Telegram, 13928, 1

Pal S., Manna A., Kale R., 2021, preprint (arXiv:2101.05267 33)

Price O., Jones G. H., Morrill J., Owens M., Battams K., Morgan H., Druckmuller M., Deiries S., 2019, Icarus, 319, 540

Rotundi A. et al., 2015, Science, 347, 6220

Samarasinha N.H., Martin M.P., Larson S.M., 2013, Cometary Coma Image Enhancement Facility, http://www.psi.edu/research/cometimen

Schleicher D.G., Farnham T.L., 2004, in Festou M. C., Keller U.,Weaver H.A., eds, Comets II. Univ. Arizona Press, Tucson, Arizona, USA, p. 449

Schleicher D.G., Knight M.M., Skiff B.A., 2020, BAAS, 52. Retrieved from https://baas.aas.org/pub/2020n6i111p05

Schulz R., McFadden L., Chamberlain A., A'Hearn M., Schleicher D., 1994, Icarus 109, 145

Schulz R., Stüwe J.A., Boehnhardt H., Gaessler W., Tozzi G.P., 2003, A\&A 398, 345

Sekanina Z., Farrell J.A., 1980, AJ 85, 1538

Vincent J.-B., 2014, Comet-toolbox: numerical simulations of cometary dust tails in your browser. Asteroids Comets Meteors conference. Helsinki

Vincent J.-B., Boehnardt H., Lara L.M., 2010, A\&A 512, A60
Waniak W., Borisov G., Drahus M., Bonev T., Czart K., Küppers M., 2009, Earth Moon Planets, 105, 327 